\title{
Fluorescence lifetime imaging in scanning microscopes: acquisition speed, photon economy and lifetime resolution
}

\author{
H. C. GERRITSEN, M. A. H. ASSELBERGS, \\ A. V. AGRONSKAIA \& W. G. J. H. M. VAN SARK \\ Debye Institute, Utrecht University, PO Box 80.000, NL 3508 TA, Utrecht, the Netherlands
}

Key words. FLIM, multiphoton microscopy, time-gating.

\section{Summary}

In this paper a detailed discussion is presented of the factors that affect the fluorescence lifetime imaging performance of a scanning microscope equipped with a single photon counting based, two- to eight-channel, time-gated detection system. In particular we discuss the sensitivity, lifetime resolution, acquisition speed, and the shortest lifetimes that can be measured. Detection systems equipped with four to eight time-gates are significantly more sensitive than the two timegate system. Only minor sensitivity differences were found between systems with four or more time-gates. Experiments confirm that the lifetime resolution is dominated by photon statistics. The time response of the detector determines the shortest lifetimes that can be resolved; about 25 ps for fast MCP-PMTs and 300-400 ps for other detectors. The maximum count rate of fast MCP-PMTs, however, is 10-100 times lower than that of fast PMTs. Therefore, the acquisition speed with MCP-PMT based systems is limited. With a fast PMT operated close to its maximum count rate we were able to record a fluorescence lifetime image of a beating myocyte in less than one second.

\section{Introduction}

Fluorescence microscopy is routinely used in biological and biophysical research. The power of fluorescence microscopy lies in the excellent contrast, selectivity and sensitivity that can be achieved with this method. The high contrast is the result of the difference between the wavelength at which the specimen is excited and the wavelength of the emitted fluorescence light (Stokes' shift). Moreover, the fluorescent molecules can be easily conjugated to chemically selective groups or antibodies, such that specific sites in a specimen can be selectively labelled. As a result it is possible to selectively

Correspondence: Hans Gerritsen, Tel. + 31302532824 ; fax: +3130 2532706; e-mail: H.C.Gerritsen@phys.uu.nl stain, for instance, specific parts of cells. This makes fluorescence microscopy a popular tool in morphological studies. The number of probes that can be distinguished in fluorescence microscopy is limited by overlap of the comparatively broad emission spectra of the fluorescent labels to two or three.

Fluorescence microscopy can also be employed for the imaging (quantification) of concentrations of molecules. A good example is the imaging of ion concentrations using fluorescent probes that alter their properties in the presence of specific ions. These probes have a high selectivity for specific ions and exhibit distinct changes in their photophysical properties in the presence of ions. Fluorescent ion-indicators can be classified into two groups: intensity probes and ratio probes. Intensity probes are less suitable for quantitative imaging due to the sensitivity for probe concentration and other intensity effects. Ratio probes are better suited for quantitative imaging experiments. However, in general these probes require an in situ calibration procedure and often the ratio probes, $\mathrm{Ca}^{2+}$ ratio probes in particular, have absorption bands in the UV. This radiation is not only potentially harmful to biological cells but also gives rise to an auto-fluorescence background in the images.

Using the fluorescence lifetime of a fluorescent molecule as a contrast mechanism can further enhance the potential of fluorescence microscopy (Draaijer et al., 1995). The fluorescence lifetime of a molecule is independent of the fluorescence intensity and differs per type of fluorescent molecule. In addition the fluorescence lifetime can be sensitive for the chemical environment of the fluorescent molecule. This property can be exploited in quantitative imaging experiments.

Fluorescence lifetime imaging (FLIM) can be used in multilabelling experiments (Sanders et al., 1995), quantitative imaging of ion-concentrations (Lakowicz \& Szmacinski, 1993), quantitative imaging of other molecules such as oxygen (Gerritsen et al., 1997), and quantification of the energy transfer efficiency in FRET experiments (Wouters \& Bastiaens, 1999). FLIM microscopes are, in general, comparatively slow. 
Acquisition times of several tens of seconds or even more are not unusual.

In this paper we will treat several practical aspects related to the acquisition speed, sensitivity and lifetime resolution of FLIM microscopes. We will limit ourselves to time-domainbased scanning FLIM microscopes.

\section{Fluorescence lifetime imaging}

Fluorescence lifetimes are generally on the order of a few nanoseconds; therefore, fast detection schemes are required for FLIM. Fluorescence lifetime imaging can be implemented using frequency domain methods (Gadella et al., 1993; Lakowicz \& Berndt, 1991, Morgan et al., 1990) and timedomain methods (Bugiel et al., 1989; Buurman et al., 1992; Schneckenburger \& Konig, 1992; Wang et al., 1991). In the former, the phase shift between the pulsed or modulated excitation light and the fluorescence is used to determine the lifetime. The phase shift between excitation and emission is introduced by the delay (fluorescence lifetime) between the emission and excitation light. This method will not be further discussed here. The time-domain method relies on the registration of the fluorescence decay after pulsed excitation. Here, usually time-gated detection or time correlated single photon counting (TCSPC)-based methods (O'Connor \& Phillips, 1984) are employed.

In the time-gated detection-based methods, the fluorescence emission is detected sequentially in two or more time gates each delayed by a different time relative to the excitation pulse (see Fig. 1). In the case of a two-gate detection scheme, the ratio of the signal acquired in the two gates is a measure of the fluorescence lifetime. For mono-exponential fluorescence intensity decays the fluorescence lifetime is given by:

$$
\tau=\Delta T / \ln \left(I_{2} / I_{1}\right)
$$

where $\Delta T$ is the time-offset between the start of the two timewindows and $I_{1}$ and $I_{2}$ are the corresponding integrated fluorescence intensities. In deriving Eq. (1) it is assumed that the two gates are of the same width. In the case of a multiexponential fluorescence decay Eq. (1) yields only an 'average' fluorescence lifetime. However, the number of time-gates can be easily extended to enable the recording of multi-exponential decays (Scully et al., 1997; de Grauw \& Gerritsen, 2001).

Time-gated detection offers the possibility of suppressing background signals correlated with the excitation pulse. Direct and multiple scattered excitation light as well as Raman scattering reaches the detector at $t \approx 0$, and can be effectively suppressed by opening the first gate a few hundred picoseconds after $t=0$. This can improve the signal-to-background ratio in the images without a significant loss of signal. Furthermore, time gating can be employed to discriminate autofluorescence in biological specimens. In general, autofluorescence has a comparatively short fluorescence lifetime
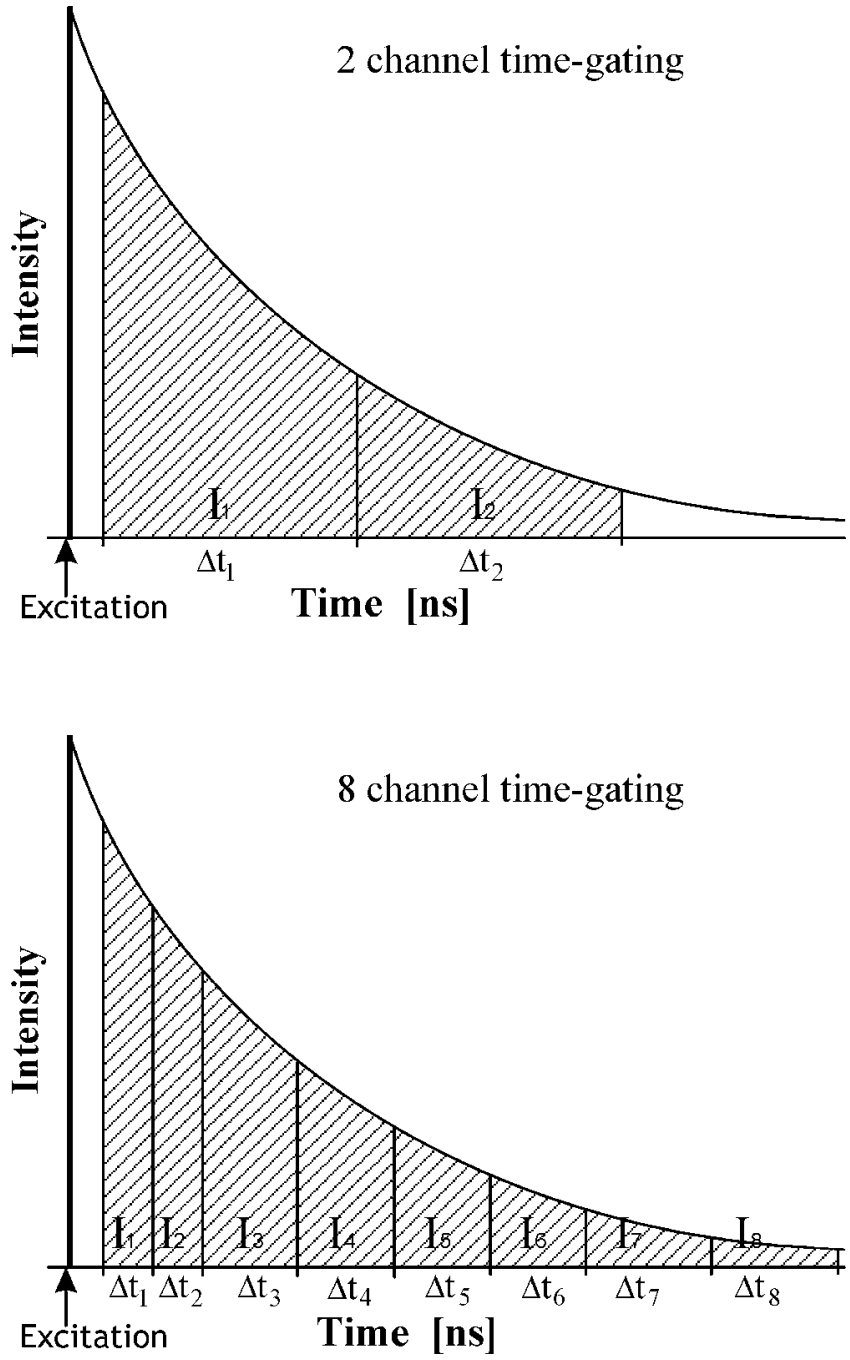

Fig. 1. Time-gated detection scheme with two and with eight time gates. After the excitation pulse the fluorescence intensity decay is captured in a number of gates. $I_{i}$ and $\Delta t_{i}$ denote, respectively, the intensity and timewidth of time gate $i$.

and the signal-to-background ratio of the images can again be improved by offsetting the first gate with respect to the excitation pulse.

We have implemented time-gated detection schemes in both a confocal microscope (Buurman et al., 1992) and in a multiphoton excitation microscope (Sytsma et al., 1998). The implementation of time-gated detection in scanning microscopes is straightforward because only one pixel is acquired at a time. In both set-ups we incorporated very efficient detection schemes that rely on the use of time-gated single photon counting, see Fig. 2.

Briefly, the pulses that are coming out of the detector (photomultiplier - PMT, MCP-PMT) or single photon counting avalanche photodiode-SPAD) are fed into a discriminator. The output of the discriminator is fed into gated fast counters that are enabled for a specific time after a preset delay 


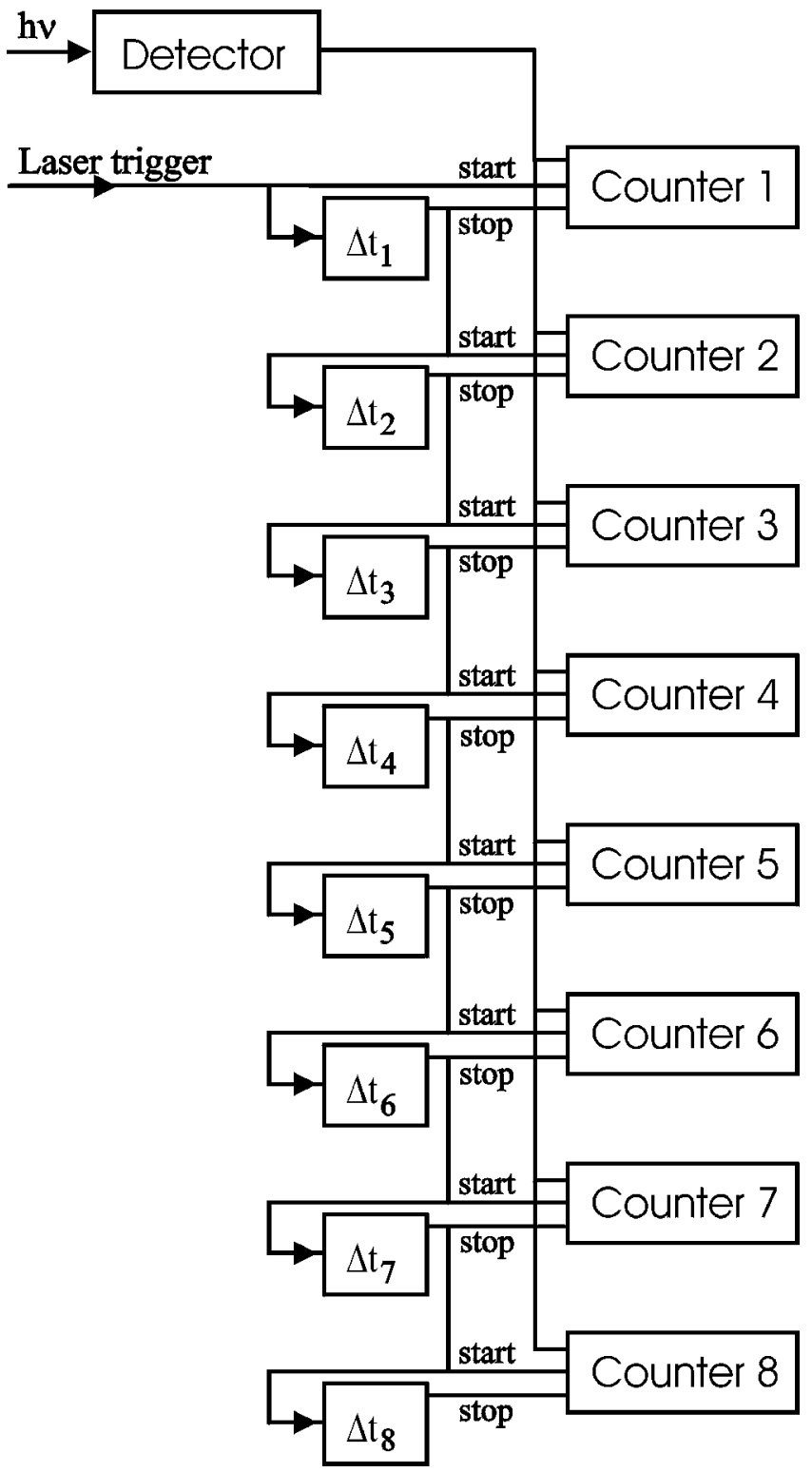

Fig. 2. The time-gated detection electronics. The pulses coming out of the detector (+ discriminator) are fed into a number of gated counters that are all sequentially enabled after every excitation pulse. The width of the gates can be set independently.

with respect to the excitation pulse. All the gates open sequentially after each and every excitation pulse. Therefore, the recorded lifetime is in general not affected by fading of the fluorescence due to photobleaching. Importantly, this approach is extremely efficient; virtually the whole decay is detected after every excitation pulse. Using single photon counting further enhances the sensitivity. In this way multiplicative noise is avoided. Moreover, the use of fast digital counters (emitter coupled logic) ensures a negligible dead time of $<0.5 \mathrm{~ns}$ of the detection electronics. Therefore, the detector unit can operate at extremely high count rates without significant pile-up. In practice, the type of detector employed limits the maximum count rate.

This FLIM module is interfaced to a PC that controls both the time offsets of the individual gates and the widths of the time-gates. The use of non-equal gate widths has several advantages. The first (early) gates are mainly sensitive for short lifetimes, whereas the later gates mainly contain signal related to longer lifetimes. Therefore, the usable lifetime range can be optimized by employing comparatively narrow early gates and wider late gates. Obviously, the total width of the gates should not exceed the period of the laser repetition frequency. For the eight time-gate unit the gate-widths can be set between 0 ns and 9530 ns. This enables the accurate and sensitive imaging of lifetimes over a broad lifetime range. Multi-exponential decays can be already measured using four (Elangovan et al., 2002) or eight (de Grauw \& Gerritsen, 2001) gates.

\section{Photon economy}

For the two-gate system Ballew \& Demas (1989) demonstrated that the optimum gate width in the case of equal gate widths amounts to 2.5 times the fluorescence lifetime. Under these conditions, the two-gate system collects the fluorescence for a time span of $5 \cdot \tau$. Assuming that the first gate opens at time zero, this corresponds to a total of $99.3 \%$ of the total fluorescence emission. This makes the method very efficient. Theoretical calculations (Kollner \& Wolfrum, 1992) and computer simulations (Gerritsen et al., 1996; de Grauw \& Gerritsen, 2001) revealed that at the optimum gate width a twogate system requires only 225 detected photons for an accuracy of $10 \%$ in the fluorescence lifetime. We demonstrated that this accuracy could indeed be realized in practice, thus proving that the accuracy is determined by photon statistics alone (Sytsma et al., 1998; de Grauw \& Gerritsen, 2001).

We investigated the photon economy of time-gated detection methods in detail (de Grauw \& Gerritsen, 2001). In particular we looked at the effect of the number of gates and the gate widths on the accuracy with which lifetimes can be determined. Briefly, in order to compare different gate settings a figure of merit is required. We employed a figure of merit, $F$, that compares the accuracy of the lifetime acquisition method with that of a simple intensity measurement, $F=(\Delta \tau / \tau) /(\Delta \mathrm{I} / \mathrm{I})$, with $\tau$ the lifetime, $\Delta \tau$ the standard deviation of the lifetime determination, $I$ the number of detected photons in the intensity measurement and $\Delta I$ the standard deviation of the intensity determination. On assuming Poisson statistics $\Delta I / I$ is proportional to $1 / \sqrt{ } I$ and the expression for $F$ can be rewritten as:

$$
F=\frac{\Delta \tau}{\tau} \cdot \sqrt{I}
$$

Now, $F$ is a measure for the sensitivity of the lifetime acquisition method that is independent of the number of counts in the decay, provided that $\Delta \tau / \tau$ is also governed by Poisson statistics. 
$F$ is always greater than one and a low value of $F$ indicates a high sensitivity.

The definition of $F$ can be used for any number of gates and enables the comparison of acquisition systems with a different number of gates and different gate settings. In the case of two gates of equal width, $\Delta \tau / \tau$ can be calculated analytically (Ballew \& Demas, 1989). However, in general this is difficult or even impossible for detection systems with more than two gates and/or gates of nonequal width. In the case that no analytical expression for $\Delta \tau / \tau$ is available, it can be obtained by means of computer simulations. We carried out such simulations for a large number of different gate settings. In the simulations random counts are accumulated in four or eight gates of arbitrary width using the probability function $P(t)=\tau \cdot \mathrm{e}^{-t / \tau}$ for the decay. From the simulated gate intensities the lifetime was determined by fitting the data with an exponential decay using a nonlinear least square Levenberg-Marquardt fit algorithm (Press et al., 1992). This procedure was repeated 2500 times to obtain the standard deviation in the lifetimes. $F$ was then calculated using Eq. (2).

The efficiencies of a number of gate settings were compared for a range of lifetimes by calculating and comparing their figures of merit. For a two-gate system with equal time-gates of $5 \mathrm{~ns}$, $F$-values were calculated analytically. Furthermore, $F$-values were calculated for a four-gate system (gate-widths 1, 1, 3, $5 \mathrm{~ns}$ ) and for two settings of an eight-gate system (gatewidths $0.5,0.5,0.5,0.5,1,1,2,4$ ns and $1,1,4,4,4,6,12$, $80 \mathrm{~ns})$. In all cases the lifetimes were obtained by fitting the simulated data, and next $F$ was calculated from the standard deviation in the lifetimes, using Eq. (2). Apart from the 1, 1, 4, $4,6,12,80 \mathrm{~ns}$ case, the total collection time per laser pulse was $10 \mathrm{~ns}$ and the delay between the excitation pulse $(t=0)$ and opening of the first gate was $0.5 \mathrm{~ns}$. This delay was added in order to account for effects related to the response time of the detection system. Typically, the response time (transit time spread) of a PMT is on the order of a few hundred ps. The choice of the gate setting is limited by the repetition frequency of the excitation source. In our set-up the maximum repetition rate of the Ti: sapphire laser amounts to $82 \mathrm{MHz}$. Consequently, the sum of the gate-widths and the delay between the excitation pulse and the opening of the first gate should be less than $12.2 \mathrm{~ns}$.

\section{Results and discussion}

\section{Time-gated fluorescence lifetime detection efficiency}

The results of the figure of merit calculations are presented in Fig. 3. The configuration with two gates has a minimum $F$ value of 1.7 at a lifetime of about $2.2 \mathrm{~ns}$. The four- and eightgate configurations have minimum $F$ values of 1.3 and 1.23 , respectively, at a lifetime of about $1.7 \mathrm{~ns}$. Using Eq. (2), the $F$ values can be converted to the number of counts (in the total decay) that is required to realize a specific accuracy,

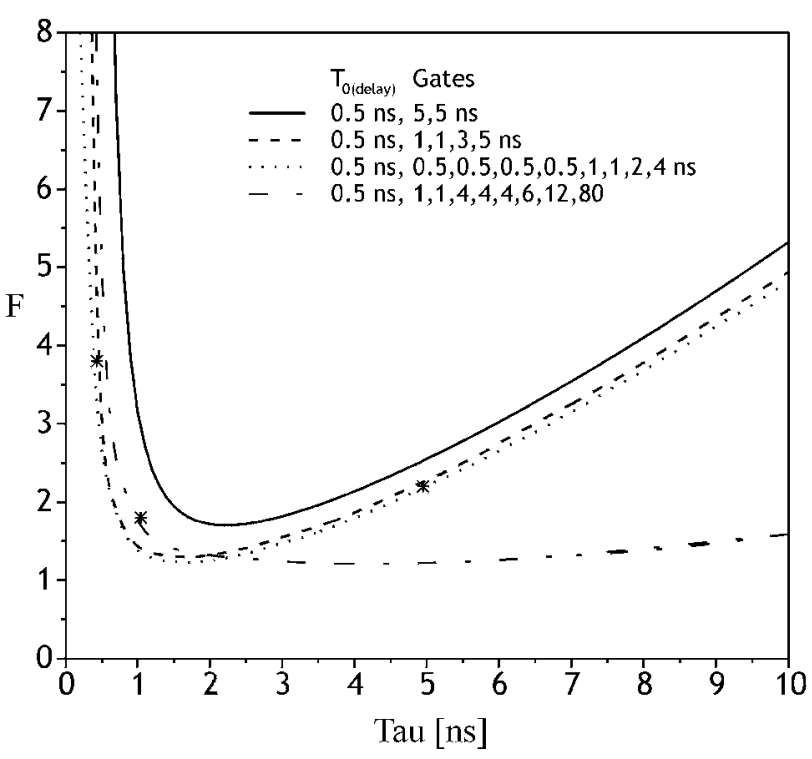

Fig. 3. The figure of merit $(F)$ curves plotted as a function of lifetime for different gate settings; two gates of $5 \mathrm{~ns}$ each, four gates of $1,1,3$ and $5 \mathrm{~ns}$, and eight gates of $0.5,0.5,0.5,0.5,1,1,2$ and 4 ns and of $1,1,4,4,4,6$, 12 and 80 ns. Low $F$ values correspond with a high sensitivity. Three experimental $F$ values, marked *, are included.

$I=(F /(\Delta \tau / \tau))^{2}$. The square of the ratio of figures of merit of two configurations is a direct measure of the relative sensitivity of these configurations at a specific $\tau, I_{1} / I_{2}=\left(F_{1} / F_{2}\right)^{2}$. Using the latter equation we find that, at the optimum lifetimes, the four- and eight-gate configurations are about twice as sensitive as the two-gate configuration. For instance, the two-gate configuration requires about 1200 counts to obtain an accuracy of $5 \%$ at the optimum lifetime value, whereas the four- and eight-gate configurations need only about 650 counts to obtain this accuracy. The curves in Fig. 3 show that the four- and eight-gate set-ups are significantly more sensitive than the two-gate set-up over the whole lifetime range. The benefit of the four- and eight-gate set-ups increases for lifetimes below 2 ns. The $F$ number of a two-gate configuration can be improved for the short lifetime range by reducing the gate-widths. However, this would reduce the sensitivity for the long lifetime range. To show the performance for longer gate-widths the $F$ numbers of simulated gates of $1,1,4,4,4,6,12$ and $80 \mathrm{~ns}$ were added to Fig. 3. These gate settings fit well with a laser repetition rate of $8.2 \mathrm{MHz}$, i.e. $1 / 10$ of the Ti: sapphire repetition rate. The performance of this setting is clearly superior for longer lifetimes (> $2.5 \mathrm{~ns})$. However, both the short lifetime performance and the minimum $F$ value remain the same.

Experimental figures of merit for erythrosin in PVA, a solution of eosin and a solution of Lucifer Yellow are also indicated in Fig. 3 (de Grauw \& Gerritsen, 2001). The lifetimes were recorded using the four-gate configuration used in the simulations. The experimental and simulated $F$ values are in good agreement. This indicates that the statistics of the 
lifetime measurements is dominated by Poisson noise and thus that the method is photon noise limited. We note that the experimental $F$ values in Fig. 3 were determined from measurements with at least several hundreds of counts per pixel integrated over all gates. Below a total of 50 counts per pixel, deviations from Poisson statistics were observed.

\section{Acquisition speed considerations}

The number of photons per pixel and the (maximum) count rate determine the acquisition speed of the fluorescence lifetime imaging system. The description of the performance of the lifetime acquisition method in terms of a figure of merit allows the calculation of the number of photons that are required in order to realize a specific lifetime resolution. The acquisition speed, however, will in general be limited by the performance of the detector. Detectors are characterized by their timing behaviour, maximum count rate and (wavelength dependent) quantum efficiency. The most common detector employed in scanning microscopy is the photo multiplier tube (PMT). Two types of PMTs exist: side-on and head-on PMTs. Side-on PMTs are often used in scanning microscopes because of their high quantum efficiency of up to $35 \%$. In practice, we found that the side-on tubes employed in scanning microscopes show poor timing properties. Transit time spreads (timing jitter) of several nanoseconds are not uncommon. This is insufficient for applications that require a subns timing resolution. At present, the best PMTs for timing critical applications seem to be head-on PMTs. Their transit time spread can be as low as several hundred picoseconds, but usually their quantum efficiency does not exceed $10 \%$. The maximum count rate that these PMTs can operate at is in general limited to 5-10 MHz. At higher count rates pulse pile-up degrades their performance and they may even be damaged when the maximum anode current is exceeded.

The shortest lifetimes that can be measured with a lifetime imaging system depend on both the timing properties of the detector and those of the detection electronics. PMTs optimized for fast timing applications may have a transit time spread as low as 300-400 ps. This limits the shortest lifetimes that can be measured with this type of tube to similar values. In order to measure shorter lifetimes, micro channel plate PMTs (MCP-PMTs) (McLoskey et al., 1996) can be used. MCPPMTs have quantum efficiencies in the order of $10 \%$ and exhibit transit time spreads as low as 25 ps, which makes them particularly suited for the measurement of fast decays. However, the MCP-PMTs are expensive and vulnerable and the maximum count rate at which they can be operated at is limited. For instance, the popular Hamamatsu R3809U-50 MCP-PMT has a maximum average anode output current of $100 \mathrm{nA}$, corresponding to a maximum count rate of several hundred $\mathrm{kHz}$. However, Hamamatsu advises operating this device up to a maximum count rate of $20 \mathrm{kHz}$.

Both PMTs and MCP-PMTs are rather poor in terms of their quantum efficiency of about $10 \%$. Alternatively one can use SPADs or PMTs with a GaAs or GaAsP photocathode. The SPADs have high quantum efficiencies, about $70 \%$ at $650 \mathrm{~nm}$, and are well suited for single photon counting applications at count rates of up to $2 \mathrm{MHz}$. Moreover, their timing jitter is specified to be about $350 \mathrm{ps,} \mathrm{which} \mathrm{makes} \mathrm{them} \mathrm{attractive}$ for lifetime imaging. The $\operatorname{GaAs}(\mathrm{P})$ photocathodes also have comparatively high quantum efficiencies of about $40-50 \%$ in the red part of the spectrum. Their maximum count rate is limited to about $1.5 \mathrm{MHz}$ and their timing resolution is about $350 \mathrm{ps}$.

Based on the above figures, we can make an estimate of the maximum frame rate that can be realized with a specific type of detector. On assuming a $256 \times 256$ pixel image and an average number of counts per pixel of 250 , we find frame acquisition times of 3.2-1.6, 640-64, 10 and $10 \mathrm{~s}$ for the PMT, MCP-PMT, SPAD and GaAS(P) PMT, respectively. Very short lifetimes of about 25 ps can only be measured with a MCPPMT, at the price of long acquisition times. Fast frame acquisition speeds in combination with a lower lifetime limit of a few hundred picoseconds can be realized with fast PMTs. Interestingly, the higher quantum efficiency detectors, SPAD and GaAS(P) PMT, combine a lower lifetime limit comparable with that of PMTs with a high quantum efficiency. This makes them interesting alternatives for low light level FLIM. The properties of the different detectors are summarized in Table 1 .

The performance of the total acquisition speed also depends on the properties of the acquisition electronics. The dead time of the acquisition electronics is particularly important. In general, large dead-times will result in the loss of counts at high count rates. For a continuous wave-like illumination the fraction of detected photons, $f$, amounts to:

$$
f=\frac{1}{1+t_{d} \cdot C_{i}},
$$

with $t_{d}$ the dead-time of the system and $C_{i}$ the incident count rate. This equation only accounts for the number of counts

\begin{tabular}{lllll}
\hline & $\begin{array}{l}\text { Max. count rate } \\
\text { in FLIM }\end{array}$ & $\begin{array}{l}\text { Time jitter } \\
(\mathrm{ps})\end{array}$ & $\begin{array}{l}\mathrm{Q}_{\max } \\
(\%)\end{array}$ & $\begin{array}{l}\text { Time/frame }(256 \times 256) \\
\text { at 250 cnts/pixel }(\mathrm{s})\end{array}$ \\
\hline PMT & $5-10 \mathrm{MHz}$ & 400 & 10 & $3.2-1.6$ \\
MCP-PMT & $25-250 \mathrm{kHz}$ & 25 & 10 & $640-64$ \\
SPAD & $2 \mathrm{MHz}$ & 350 & 70 & 10 \\
GaAs(P) PMT & $1.5 \mathrm{MHz}$ & 350 & 45 & 10 \\
\hline
\end{tabular}

Table 1. Summary of the properties of different types of detectors that can be used for (time domain) fluorescence lifetime imaging. 
lost due to pile-up. In lifetime imaging the probability of the incident fluorescence light on the detector usually follows a (multi)exponential distribution. Therefore, the probability of pile-up is non-uniform in time and peaks at $t=0$ of the exponential function. Consequently, when pile-up takes place the recorded fluorescence decay is distorted and the fitted lifetimes show a count rate dependency. In our experience, a pile-up of 5-10\% does not significantly affect the recorded fluorescence lifetimes. For a detection system with a dead time of $10 \mathrm{~ns}$ this corresponds to a maximum count rate of 5-10 MHz. This decreases to $250-500 \mathrm{kHz}$ for a detection system with a dead time of 200 ns.

In TCSPC (lifetime spectroscopy) experiments that require the best possible lifetime resolution and reproducibility, the pile-up should be less than 1\% (Demas, 1983; O'Connor \& Phillips, 1984). This requires maximum count rates of $1 \mathrm{MHz}$ and $50 \mathrm{kHz}$ for the systems with $10 \mathrm{~ns}$ and the $200 \mathrm{~ns}$ dead time, respectively.

The time-gated detection system employed here has a dead time of less than $0.5 \mathrm{~ns}$. This is much shorter than the dead times of the PMTs and other detectors and does not affect the maximum count rate capability of the lifetime imaging system. In contrast, more conventional time-correlated single photon counting-based systems usually have dead times in the order of $200 \mathrm{~ns}$. The effect of this dead time on the maximum count rate capability of a lifetime imaging system is much more severe than the maximum count rate capability of PMTs.

High FLIM acquisition rates can indeed be realized in practice. With our module we have been able to acquire images at pixel rates in the order of $125 \mathrm{kHz}$ (i.e. a $8 \mu$ pixel $^{-1}$ dwell time). This was realized using a Hamamatsu R1894 PMT and a Hamamatsu H5783P PMT module, both in combination with the time-gating electronics described above. Realizing high frame rates in FLIM is much more demanding than in fluorescence intensity imaging. In FLIM, fluorescence signal levels are required at least one order of magnitude higher than in fluorescence intensity imaging. Therefore, high quantum efficiency detectors such as SPADs and the novel GaAs(P) photo cathode PMTs are potentially interesting for lifetime imaging.

As an example of fast lifetime imaging we recorded intensity and fluorescence lifetime images of Indo-1 stained rat myocytes (see Fig. 4). The myocytes spontaneously beat at rates of $0.5-2 \mathrm{~Hz}$ at $37^{\circ} \mathrm{C}$ and the beating was accompanied by large $\mathrm{Ca}^{2+}$ fluxes (Berlin \& Konishi, 1993). The images were recorded in a homemade two-photon excitation microscope (Sytsma et al., 1998) at an excitation wavelength of $700 \mathrm{~nm}$. Indo-1 shows different fluorescent lifetimes for the free probe and the ion-bound probe of about 1.4 and $1.66 \mathrm{~ns}$, respectively (Lakowicz \& Szmacinski, 1993). Despite the comparatively low lifetime contrast between the free and bound forms of the dye, lifetime images $(256 \times 256$ pixels $)$ could be recorded at a rate of 1 frame $\mathrm{s}^{-1}$. Here, the R1894 PMT was
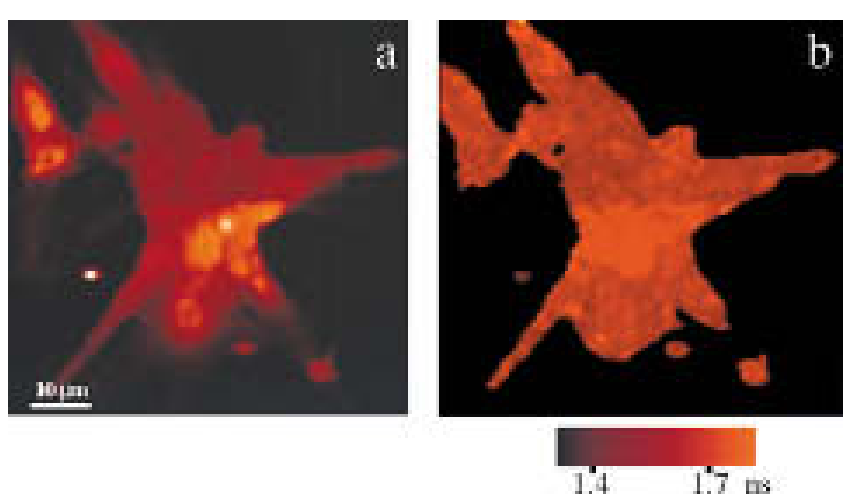

Fig. 4. Intensity (a) and lifetime (b) images of an Indo-1 stained rat myocyte. The banding in the images is due to the beating of the myocytes. The $256 \times 256$ pixel images were recorded simultaneously in $1 \mathrm{~s}$.

used at an average count rate of approximately $10 \mathrm{MHz}$. Both the intensity, Fig. 4(a), and lifetime image, Fig. 4(b), were median filtered. The edges of the lifetime image are sharper due to thresholding. Lifetimes were only calculated for pixels containing more than 50 counts. Pixels with fewer counts were set to zero. The calibration bar below the lifetime image gives an indication of the lifetimes. No attempt was made to convert the lifetimes into calcium concentrations. Both the intensity and the lifetime image show bands due to the beating of the myocyte during image acquisition. The myocyte beat about twice during the image acquisition.

\section{Conclusions}

The detectors currently available for FLIM in scanning microscopes yield minimum frame acquisition times that vary between about $1 \mathrm{~s}$ and more than $1 \mathrm{~min}$. Lifetimes in excess of $0.3-0.5 \mathrm{~ns}$ can be very well imaged using fast PMTs. Shorter lifetimes require the use of MCP-PMTs at the expense of low acquisition rates. By running conventional fast PMTs close to the maximum output current it is possible to record lifetime images from a biological specimen at a rate of about 1 frame $\mathrm{s}^{-1}$.

\section{Acknowledgements}

This work is supported by the European Community, grant QLRT/1999-01340, and by the Dutch Technology Foundation (STW).

\section{References}

Ballew, R.M. \& Demas, J.N. (1989) An error analysis of the rapid lifetime determination method for the evaluation of single exponential decays. Anal. Chem. 61, 30-33.

Berlin, J. \& Konishi, M. (1993) $\mathrm{Ca}^{2+}$ transients in cardiac myocytes measured with high and low affinity $\mathrm{Ca}^{2+}$ indicators. Biophys. J. 65, 1632-1647. 
Bugiel, I., König, K. \& Wabnitz, H. (1989) Investigation of cells by fluorescence laser scanning microscopy with subnanosecond time resolution. Laser Life Sciences, 3, 1-7.

Buurman, E.P., Sanders, R., Draaijer, A., Gerritsen, H.C., Van Veen, J.J.F., Houpt, P.M. \& Levine, Y.K. (1992) Fluorescence lifetime imaging using a confocal laser scanning microscope. Scanning, 14, 155-159.

Demas, J.N. (1983) Excited State Lifetime Measurements. Academic Press, New York.

Draaijer, A., Sanders, R. \& Gerritsen, H.C. (1995) Fluorescence lifetime imaging, a new tool in confocal microscopy. Handbook of Biological Confocal Microscopy (ed. by J. Pawley), pp. 491-505. Plenum, New York.

Elangovan, M., Day, R.N. \& Periasamy, A. (2002) Nanosecond fluorescence resonance energy transfer-fluorescence lifetime imaging microscopy to localize the protein interactions in a single living cell. J. Microsc. 205, 3-14.

Gadella, T.W., Jovin, T.M. \& Clegg, R.M. (1993) Fluorescence lifetime imaging microscopy (FLIM) - spatial resolutions of microstructures on the nanosecond time scale. Biophys. Chem. 48, 221-239.

Gerritsen, H.C., Sanders, R., Draaijer, A. \& Levine, Y.K. (1996) The photon economy of fluorescence lifetime imaging. Scanning, 18, 5556.

Gerritsen, H.C., Sanders, R., Draaijer, A. \& Levine, Y.K. (1997) Fluorescence lifetime imaging of oxygen in living cells. J. Fluoresc. 7, $11-16$.

de Grauw, C.J. \& Gerritsen, H.C. (2001) Multiple time-gate module for fluorescence lifetime imaging. Appl. Spectrosc. 55, 670-678.

Kollner, M. \& Wolfrum, J. (1992) How many photons are necessary for fluorescence-lifetime measurements? Chem. Phys.Lett. 200, 199204.

Lakowicz, J.R. \& Berndt, K.W. (1991) Lifetime-selective fluorescence imaging using an Rf Phase-sensitive camera. Rev. Sci. Instrum. 62, $1727-1734$.
Lakowicz, J.R. \& Szmacinski, H. (1993) Fluorescence lifetime-based sensing of $\mathrm{Ph}, \mathrm{Ca}^{-2(+)}, \mathrm{K}^{+}$and glucose. Sens. Actuator B - Chem. 11, 133143.

McLoskey, D., Birch, D.J.S., Sanderson, A., Suhling, K., Welch, E. \& Hicks, P.J. (1996) Multiplexed single-photon counting.1. A time-correlated fluorescence lifetime camera. Rev. Sci. Instrum. 67, 2228-2237.

Morgan, C.G., Mitchell, A.C. \& Murray, J.G. (1990) Nano-second timeresolved fluorescence microscopy: principles and practice. Trans. Roy. Microsc. Soc. 463-466.

O'Connor, D.V. \& Phillips, D. (1984) Time-Correlated Single Photon Counting. Academic Press, London.

Press, W.H., Teukolsky, S.A., Vettering, W.T. \& Flannery, B.P. (1992) Numerical Recipes in C: the Art of Scientific Computing. Cambridge University Press, Cambridge.

Sanders, R., Draaijer, A., Gerritsen, H.C. \& Levine, Y.K. (1995) Selective imaging of multiple probes using fluorescence lifetime contrast. Zool. Stud. 34, 173-174.

Schneckenburger, H. \& Konig, K. (1992) Fluorescence decay kinetics and imaging of $\mathrm{Nad}(\mathrm{P}) \mathrm{H}$ and flavins as metabolic indicators. Opt. Eng. 31, $1447-1451$.

Scully, A.D., Ostler, R.B., Phillips, D., O’Neill, P.O., Townsend, K.M., Parker, A.W.\& MacRobert, A.J. (1997) Application of fluorescence lifetime imaging microscopy to the investigation of intracellular PDT mechanisms. Bioimaging, 5, 9-18.

Sytsma, J., Vroom, J.M., De Grauw, C.J. \& Gerritsen, H.C. (1998) Time-gated fluorescence lifetime imaging and microvolume spectroscopy using two-photon excitation. J. Microsc. 191, 39-51.

Wang, X.F., Uchida, T., Coleman, D.M. \& Minami, S. (1991) A 2dimensional fluorescence lifetime imaging-system using a gated image intensifier. Appl. Spectrosc. 45, 360-366.

Wouters, F.S. \& Bastiaens, P.I.H. (1999) Fluorescence lifetime imaging of receptor tyrosine kinase activity in cells. Curr. Biol. 9, 1127-1130. 